\title{
Implementation of Digital Twins for the Analysis of Test Programs of Technical Systems
}

\author{
Olga Isaeva, Nikita Kulyasov and Sergey Isaev \\ Institute of Computational Modelling of the Siberian Branch of the Russian Academy of Sciences, 50/44 \\ Akademgorodok, Krasnoyarsk, 660036, Russia, Krasnoyarsk, Russia
}

\begin{abstract}
This article presents a method for the analysis of test programs for informational interaction of technical systems with the command-and-software control. The study also deals with the creation of formal principles of the analysis of the structure and knowledge base of the intellectual simulation model, as well as it considers the tools for their infographic presentation. The new method is based on building and using digital twins combining the software-and-mathematical models of devices and knowledge bases describing the principles of their functioning and retrospective and operational data acquired during testing of the real equipment. The stages of the method implementation include the construction of digital twins of the function of the tested object, automated building of the knowledge base from the test programs, comparison of the constructed knowledge bases, simulation of environmental systems during autonomous tests and analysis of the informational interaction of the tested devices. The knowledge base is built on the basis of the parameters set in the test programs determining commands, ways of data transmission, tickets and actions of the onboard systems. The rules of the knowledge bases are simply interpreted, clear and they are described in terms of the subject area. The method provides the consideration of the existing variety of methods for the onboard equipment control performed on the basis of different approaches and communication protocols which can be set in the model during the onboard equipment design.
\end{abstract}

\section{Keywords}

Digital twin, modeling, technical systems, tests, knowledge base, infographic

\section{Introduction}

Modern industrial production is now at the stage of digital transformation. The concept of digital twins plays a significant role in it. Its purpose is to provide the transition from traditional designing and iterative revision of technical systems based on expensive multiple full-scale tests to the analysis of critical conditions of products with the help of digital models. M. Grieves [1] is the one who came up with the idea of a digital twin which is a reflection of the integration of historical and actual data on a physical object or a process and methods of their acquisition, transformation and application in order to increase the production efficiency. Digital twins allow studying devices in the process of their development during specified observation intervals, checkpoints, acceptable parameter limits and examining the processes which cannot be reproduced on real objects [2].

Russian and foreign researchers successfully apply the models for the analysis of the operation of technical objects. The control of onboard equipment failures is per-formed using the model presenting matrices of condition vectors and a sequence of the control actions [3]. For radio line monitoring, models of spatial accessibility of radio emissions in the command-and-relay systems are built [4].

SibDATA 2021: The 2nd Siberian Scientific Workshop on Data Analysis Technologies with Applications 2021, June 25, 2021, Krasnoyarsk, Russia

EMAIL: isaeva@icm.krasn.ru (O. Isaeva)

ORCID: 0000-0002-5061-6765 (O. Isaeva); 0000-0001-5582-9498 (N. Kulyasov); 0000-0002-6678-0084 (S. Isaev)

(c) (i) 2021 Copyright for this paper by its authors.

Use permitted under Creative Commons License Attribution 4.0 International (CC BY 4.0)

CEUR Workshop Proceedings (CEUR-WS.org) 
Prognosis of the quality indicators of onboard systems and consideration of external influence factors is made by examining fuzzy borders of the working conditions of the onboard equipment [5].

For the testing support of complex technical systems, we suggest a set of methods providing the construction and use of digital twins which combine software-and-mathematical models of the devices, knowledge base of their operating principles, retrospective and operational data acquired during the testing of real equipment [6]. These methods provide the construction of test procedures, extension of the measurement functions, control of the command-and-telemetry interaction of the onboard systems, etc. The methods were tested during the study of the functioning of spacecraft onboard systems.

The purpose of our research is to create a method allowing the analysis of the completeness of the tests conducted for the informational interaction of the technical systems with the command-andsoftware control, on the basis of a digital model of their functioning. The study also deals with the creation of formal principles of the analysis of the structure and knowledge base of the intellectual simulation model, as well as it considers the tools for their infographic presentation. The new method will increase the quality of preparing and conducting the tests and will reduce their cost.

The method of test program analysis includes the following stages: 1. Construction of digital twins of the function of a tested object, which combine knowledge bases, software-and-mathematical models and data the full-scale tests; 2. Automated build-ing of a knowledge base from the test programs; 3. Comparison of the constructed knowledge bases with regard to the specified analysis criteria. We suggest formalization of the method providing its constructability - performability with the help of infographic tools, and interoperability - the ability to be built-in into the existing scheme of conducting tests.

\section{Method of test program analysis}

\subsection{Formalization of the test task of onboard systems}

Generally, the process of testing includes the control impacts performed on the tested object, and monitoring and analysis of the data appearing as a result of such actions [7]. The test scheme can be found in figure 1.

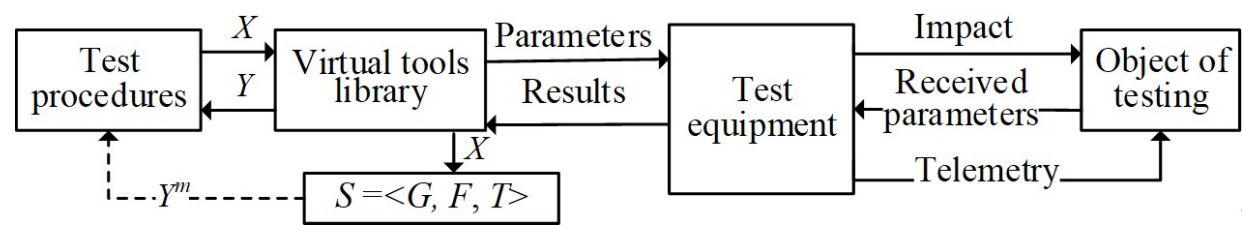

Figure 1: Scheme of conducting a test

The object of control is provided as a function of transformation of the input variables into the output ones with the set limit conditions and acceptable changes in the parameters and the measured values: $O=<G^{o}, Y=F^{o}(X, T)>$, where $O$ is the object of testing; $G^{o}$ is the structure of informational interaction; $F^{o}$ is the function setting consistency of the input variables $X$ with the output ones $Y$, at time $T$. For transmitting the control actions, the control-and-verification equipment and virtual tools library are used, providing the interaction with the object of control. The task of testing is to demonstrate that $\forall x \in X \exists y \in Y \mid y \in\left[y^{*}-\Delta y, y^{*}+\Delta y\right]$, where $y^{*} \in Y^{*}$ is the number of reference values, $\Delta y$ is the measurement tolerance. If $\exists y \notin\left[y^{*}-\Delta y, y^{*}+\Delta y\right]$, then $y$ is the unacceptable value of the testing results.

A digital twin is an intellectual model which simulates the behavior of the object of control and paired devices. The model $S=<G, F, T>$, where $G$ is the structure-and-parametric embodiment of the model, $F$ is the methods of functioning, $T$ is the time moments which allow obtaining the output results $Y^{m}$ on the basis of the initial data $X^{0}$ and input parameters $X^{i}$. The model has the structure $G=<B, I, C, D, P>$, where $B=\cup B_{i}$ are the elements, $I=\cup I_{q}^{i}$ are the interfaces, $C=\cup C^{i j}{ }_{n l}$ are the connections of the model elements, $C^{i j}{ }_{n m}=<I_{n}^{i}, P_{l}>$ is the connection of the elements $B_{i}$ and $B_{j}$ via the interfaces $I_{n}^{i}$ and $P_{l}, D$ are the data structures, $P=X \cup Y$ are the input and output parameters. The methods of the model operation: $F=\{R: A \rightarrow Z, Y=V(X)\}$, where $R: A \rightarrow Z$ is the the set of the rules of 
the knowledge base, $A$ is the rule antecedent, $Z$ is the consequent, $Y=V(X)$ are the software-andmathematical models. The test task in this case is to demonstrate that $\forall x \in X \exists y \in Y \mid y \in\left[y^{m}-\Delta y\right.$, $y^{m}+\Delta y$ ], where $y^{m} \in Y^{*}$ are the results of simulation. The description of the model and test methods on its basis are provided in $[8,9]$.

\subsection{Creation of the knowledge base on the basis of the test programs}

Building the knowledge base is performed on the basis of the parameters set in the test programs determining commands, ways of data transmission, tickets and actions of the onboard systems, criteria of control for the testing results. The chosen way of formalization of the knowledge base rules complies with the methods of the expert discussion. It is simply interpreted, clear and described in terms of the subject area.

For each command $k \in K \subseteq X$, for which the test procedures are determined, the rules of the type $R$ : $A \rightarrow Z$ are built, where $A \subseteq<I, X, T>, Z \subseteq<I, Y, T>$ :

1. The rule of command acceptance $R_{2}: A_{21}\left(I_{2}, x_{1}\right) \cup A_{22}\left(x_{1}\right) \rightarrow Z_{2}(k)$, where $A_{2}=$ «byte array $x_{1}$ has arrived to the interface $I_{2} \in C^{k} », A_{22}=\left\langle\right.$ structure $x_{1}=D^{k} », Z_{2}=\left\langle k=\right.$ byte array $x_{1} »$, where $I_{2}$ is the interface specified in the test procedure as an accepting one for the object of control, $D^{k}$ is the structure of the command packages (which are formed during the description of the test procedures; they are different for different devices).

2. The rule of command transmission to the onboard control complex $R_{3}: A_{3}(k) \rightarrow Z_{3}\left(I_{3}, k\right)$, where $A_{3}=$ «Type $k », Z_{3}=\left\langle\right.$ transmit $k$ to interface $I_{3} \in C^{r} »$, where type $k$ returns the type of the command by which the processing device is determined.

3. The command receipt rule $R_{4}: A_{41}\left(I_{3}, x_{2}\right) \cup A_{42}(k) \rightarrow Z_{4}\left(I_{4}, k\right)$, where $A_{41}=\ll$ «the byte array $x_{2}$ has arrived to the interface $\left.I_{3} \in C^{r}\right\rangle, A_{42}=\left\langle\right.$ the structure of the array $\left.x_{2}=D^{k}\right\rangle, Z_{4}=$ «send data $D R_{k}$ to the interface $I_{4} \in C^{r} \gg$, where $C^{r}$ are the data exchange interfaces between the onboard systems.

4. The telemetry transmission rule $R_{5}: A_{51}\left(I_{5}, x_{3}\right) \cup A_{52}\left(x_{3}\right) \rightarrow Z_{4}\left(I_{5}, T M_{j}\right)$, where $A_{4}=\langle$ the byte array $x_{3}$ has arrived to the interface $I_{5} \in C^{t} », A_{52}=\left\langle\right.$ structure of the array $\left.x_{3}=D^{t}\right\rangle, Z_{5}=\left\langle\left\langle T M_{j}=\right.\right.$ $x_{3} \gg, T M_{j} \in T M$ is the telemetry, $j$ is the the number of the parameter in the telemetry frame.

5. The set of the rules of command execution and telemetry control: $R_{l}{ }^{k}: A_{l}\left(T M_{j}\right) \rightarrow Z_{l}($ Res $)$ $(l=1, \ldots,|\operatorname{Cont}(k)|)$, where $|\operatorname{Cont}(k)|$ is the number of conditions for the command execution control $k$, specified in the test procedures, $j$ is the the number of the parameter in the telemetry frame, $A_{l}\left(T M_{j}\right)=\left\langle\right.$ parameter $T M_{j}$ at the address $\left.A d r_{l}=\operatorname{Res} \mid\right\rangle, Z_{l}=\langle\langle$ telemetry control $\left.T M_{j}\right\rangle$.

The knowledge base shows the specific of the tested equipment operation and it is used for the analysis of test program completeness.

\subsection{Creation of the knowledge base on the basis of the test programs}

Let us define the knowledge base built from the test programs as $R_{N}$ and the reference knowledge base as $R_{E}$. The reference knowledge base can be a base built by an expert in the subject area during the design of onboard equipment or it can be automatically created on the basis of the working methods of operationally ready equipment. For the research, it is necessary to perform the following sequence of actions:

1. Create command bases $K_{N}=\operatorname{Pr}\left(R_{N}, K\right)$ from the test programs and $K_{E}=\operatorname{Pr}\left(R_{E}, K\right)$ from the reference knowledge base, where $P r$ is the projection function, and prove that $K_{N}=K_{E}$.

2. Choose rules for each command: $R_{N}=\operatorname{Sel}\left(R_{N}, k \in K_{N}\right), R_{E}=\operatorname{Sel}\left(R_{E}, k \in K_{E}\right)$, where $\operatorname{Sel}$ is the function of selection by a set condition, and prove that $R_{N}=R_{E}$ for the object of the control simulator.

3. For each element of the model $B_{i}$, build chains of the rules $R c h_{N}{ }^{i} \subseteq R_{N}{ }^{\prime}$ for which $\operatorname{Dep}\left(R c h_{N}{ }^{i}, R c h_{N}^{j}\right)$ is true, and $R c h_{E}{ }^{j} \subseteq R_{E}$ for which $\operatorname{Dep}\left(R c h_{E}{ }^{i}, R c h_{E}{ }^{j}\right)$, where $R c h$ is the chain of the rules completed in the process of the logical output. Dep is the relation which 
defines that the completion of one rule chain $R c h^{i}$ entails the completion of another rule chain $R c h^{j}$ in the logical output.

4. Unite the rule chains built for the element simulating the object of control with the dependent rule chains of the knowledge base. $\operatorname{FMod}_{N}\left(B_{1}, \ldots, B_{p}\right)=\operatorname{Rch}_{N}{ }^{1} \cup \ldots \cup \operatorname{Rch}_{N}{ }^{p}$, so that $\operatorname{Dep}\left(\operatorname{Rch}_{N}{ }^{i}, R c h_{N}^{j}\right)$ or $\operatorname{Dep}\left(R c h_{N}^{j}, R_{c h}{ }^{i}\right)$ are completed, where $p=|B|, B_{i}$ is the object of control, $B_{j}(j \in[1, p])$ is the paired device or surrounding system. Also, build $F_{M o d}\left(B_{1}, \ldots, B_{p}\right)$ under the condition that $\operatorname{Dep}\left(\operatorname{Rch}_{E}^{i}, \operatorname{Rch}_{E}{ }^{j}\right)$ or $\operatorname{Dep}\left(\operatorname{Rch}_{E}{ }^{j}, \operatorname{Rch}_{E}{ }^{i}\right)$.

5. Compare the set $F_{M o d}\left(B_{1}, \ldots, B_{p}\right)$ with the set $F_{M o d}\left(B_{1}, \ldots, B_{p}\right)$ and find an error.

The formalization allows automation of the analysis of the test program completeness for the object in the case where there exists a simulation model of the object functioning and its working methods are defined in the knowledge base. Our approach is also applicable when there is no reference model. In this case, the test program completeness analysis is made for similar devices of the main and backup equipment. As a reference knowledge base, a set of rules for the functioning of the main equipment is chosen and they are compared with the rules by which the backup kits are tested. The command execution logic and interaction via switching interfaces are tested.

\section{The implementation results of the method of the test program analysis}

The results of the method implementation are infographic tools for the test program analysis. We introduce a graphical demonstration of the comparison results of the knowledge base built on the basis of the test procedures with the reference knowledge base created during the design of the onboard system and reflecting the behavior of the devices specified in the technical documentation. An example of the comparison of the knowledge bases for the object of control, i.e. the commandand-measurement system of a spacecraft is presented in Fig. 2.

The infographic elements demonstrating the matching nodes are the following: pictograms $\mathbf{O}$, nodes containing the rules of the reference base which do not exist in the base built from the test procedures $\mathbb{(})$, nodes containing excessive actions in the testing methods $\mathbb{0}$, lines which are the transitions between the logical elements of the model in the process of logical output. The following notations are used in the figure: TM request is the onboard equipment working mode when telemetry information packages are collected, formed and transmitted to the ground segment, OCS CU is the onboard control complex, CCU is the interface module of the command and measurement system, ODGS is the onboard remote signaling equipment, TRANS is the transmitter, RECIV is the receiver.

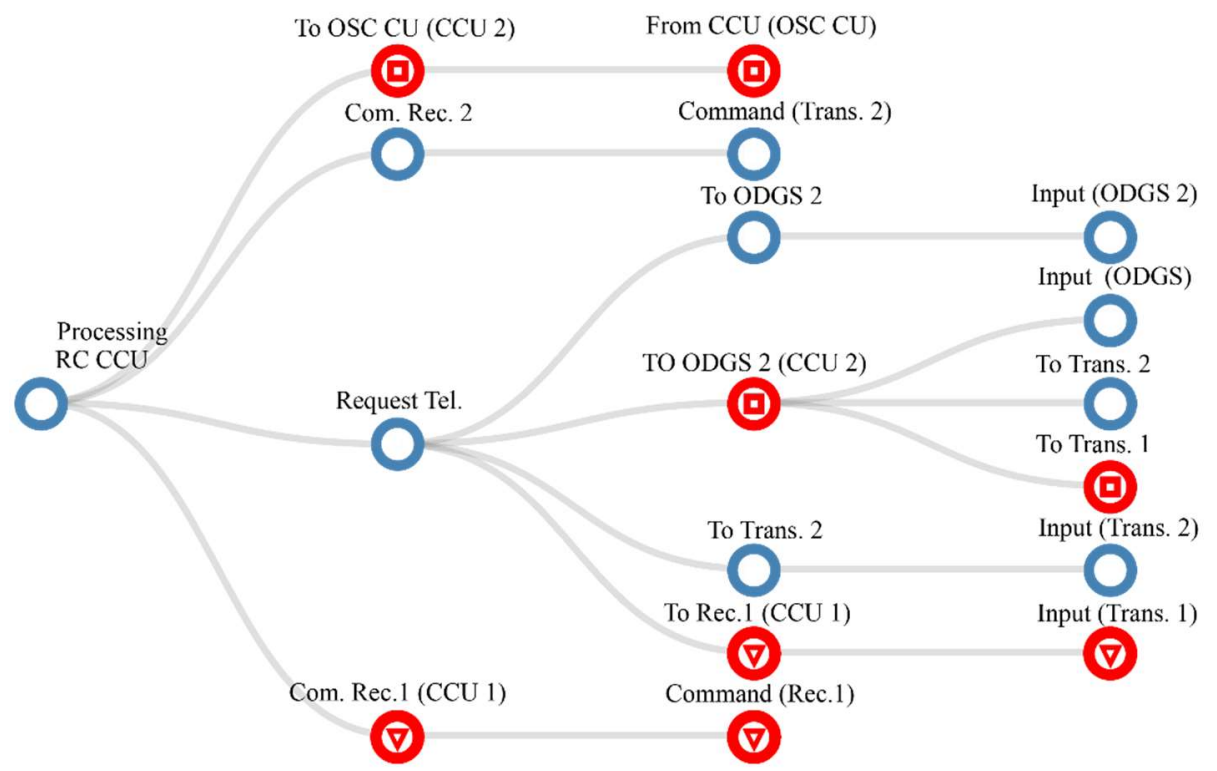

Figure 2: Visual presentation of the analysis results of the test programs 
When there is no reference knowledge base, the method allows the analysis of the test program completeness for similar devices of the main and backup equipment. An example of the comparison of the test programs in this case is shown in figure 3.

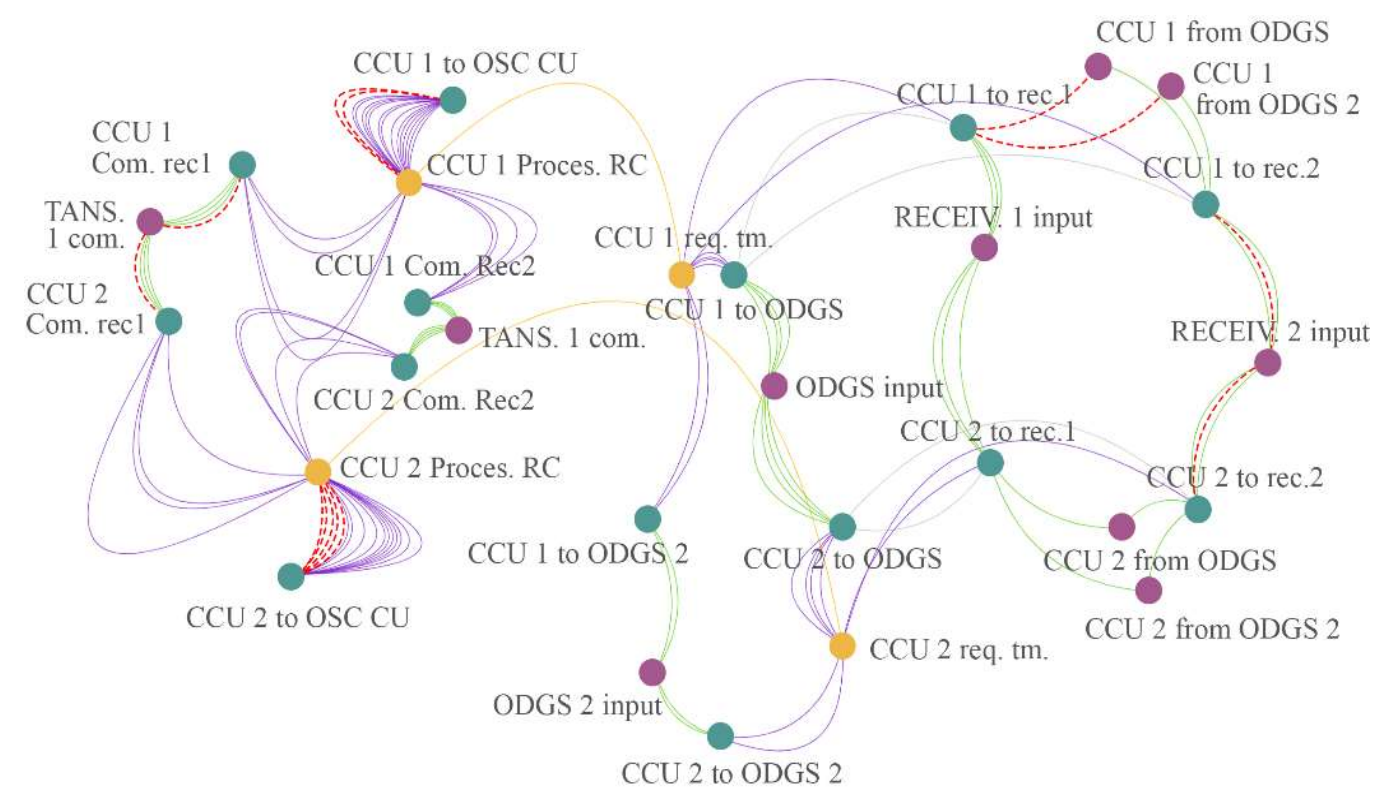

Figure 3: Visual presentation of the test programs for the main and backup equipment

For comparison, a set of the main equipment operating rules is taken as a reference base. The test actions, lacking for the backup kit, are denoted by the dotted line.

The analysis result is a list of errors reflecting the commands and the test methods not complying with the reference knowledge base. An example of the error list is provided in Figure 3.

\section{Comparison of logical structures}

\begin{tabular}{|c|c|c|c|}
\hline \multirow{2}{*}{$\begin{array}{l}\text { Chain } 1 \text { (Start elements) } \\
\text { Element from first chain }\end{array}$} & \multicolumn{3}{|c|}{ Chain 2 (Start elements) } \\
\hline & missed logic & Element from second chain & missed logic \\
\hline $\mathrm{CCU}_{1}$ (request Tm) & & CCU 2 (Request Tm) & \\
\hline To ODCS & There is no transfer rule & To Trans. 1 & There is no transfer rule \\
\hline To Trans. 1 & There is no transfer rule & Input (Trans. 1) & There is no receiver rule \\
\hline Input (Rec. 1) & There is no receiver rule & & \\
\hline
\end{tabular}

Figure 4: Error list

The error table of the knowledge base created on the basis of the tests contains the lists of the model elements with the rules lacking in the reference knowledge base or in the created one.

\section{Discussing the results and possibilities}

The approaches of digital twins combining the knowledge bases, software-and-mathematical models and results of the full-scale tests for the analysis of the operationally ready equipment are highly perspective. In this format, for preparing and conducting tests, they provide the consideration of the existing variety of methods for the onboard equipment control performed on the basis of different approaches and communication protocols which can be set in the model during the onboard equipment design. The automated comparison of the operating methods of the model presenting the 
design solutions with the model built automatically on the basis of the object of control testing data allows making a conclusion about the completeness of the test programs. If necessary, the test programs can be automatically expanded on the basis of the digital twin data.

This approach can be used in the studies of test programs for similar devices of the main and backup equipment. As a reference base, a subset of rules of the main equipment operation is chosen. The visual infographic tools automate the analysis of the test procedures in order to provide the completeness of the study both of the physical characteristics of the devices and of the logic of their interaction with the onboard equipment and ground segment, which increases the quality of creating digital twins for the spacecraft onboard systems.

It is possible to extend this approach to analyze the functioning of high-tech products such as, for example, industrial complexes built within the technology of goods web, "smart house" and other systems with the command-and-software control. The approach allows building and implementation of industrial knowledge bases, integrating the subject area expertise and the data of the technical system testing.

\section{Conclusion}

The implementation of digital twins for the creation of knowledge bases on the basis of test programs and analysis of the created models in comparison with the reference knowledge bases reflecting the design solutions, allows the automation of preparing the spacecraft onboard system testing. The test program analysis is very important for the onboard equipment off-line testing since it is necessary to consider all the possible ways of informational and telecommunication interaction of an object of control with the paired devices while they are not physically connected. Digital twins are used for automatic building of test programs. They simulate the functions of paired systems which aids in reducing labor costs and increases the quality of the analysis of informational interaction between the tested devices.

\section{Acknowledgments}

This work is supported by the Krasnoyarsk Mathematical Center and financed by the Ministry of Science and Higher Education of the Russian Federation in the framework of the establishment and development of regional Centers for Mathematics Research and Education (Agreement No. 075-022021-1384)

\section{References}

[1] M. Grieves, J. Vickers: Digital twin: mitigating unpredictable, undesirable emergent behavior in complex systems, URL: https://www.researchgate.net/publication/307509727_Origins_ of the Digital_Twin_Concept.

[2] E. Glaessgen, D. Stargel,The Digital Twin Paradigm for Future NASA and U.S. Air Force Vehicles. In: 53rd Structures, Structural Dynamics, and Materials Conference: Special Session on the Digital Twin, Honolulu, Hawaii, 2012, pp. 1-14.

[3] A. Tyugashev, An approach to ensuring fault tolerance of spacecraft based on design automation of intelligent onboard software, Reliability and quality of complex systems 2(14), (2016) 9-16.

[4] A. Andreev, V. Khatsyuk, Algorithm for assessing the spatial availability of radio emissions from spacecraft of command and relay systems using simulation modeling, Proceedings of the Military Space Academy A.F. Mozhaisky 650 (2016) 57-61.

[5] A. Mironov, E. Mironov, O. Shestopalova, Predicting the quality of functioning of spacecraft onboard equipment in conditions of fuzzy information about the boundaries of the area of operable states, Information and economic aspects of standardization and technical regulation 4 (32) (2016) 1-10. 
[6] O. Isaeva, Technology of spacecraft on-board equipment design support. IOP Conf. Series: Materials Science and Engineering, volume 1047, 2021, 012115. doi: 1047012115doi:10.1088/1757-899X/1047/1/012115.

[7] G. Rannev, Measuring information systems. M., Publishing Center "Academy”, 2010.

[8] L. Nozhenkova, O. Isaeva, A. Koldyrev, Creation of the base of a simulation model's precedents for analysis of the spacecraft onboard equipment testing results equipment, Advances in Intelligent Systems Research 151, (2018) 78-81. doi: 10.2991/cmsa-18.2018.18.

[9] O. Isaeva, N. Kulaysov, Isaev S. Method of structural and graphical analysis and verification of intellectual simulation model, Tomsk state university journal of control and computer science 50 (2020) 79-88. doi: 10.17223/19988605/50/10. 